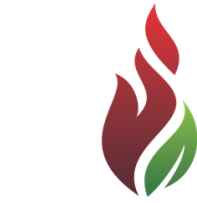

SUSTENERE

Publishing Corporation

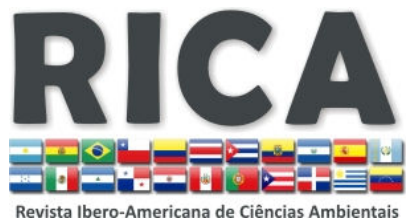

Journals Homepage:

www.sustenere.co/journals

\section{REVISÃO INTEGRATIVA SOBRE APLICAÇÃO DAS METODOLOGIAS DE AVALIAÇÃO DE RISCOS À SAÚDE E AO AMBIENTE}

\section{RESUMO}

É consenso, na literatura, que não existe uniformização de metodologias e processos para avaliação de risco na área da saúde e do meio ambiente. Neste trabalho foi realizada uma ampla revisão da produção científica nacional e internacional sobre a aplicação das principais metodologias de avaliação de risco. É apresentada uma análise comparativa detalhada do processo avaliativo de risco, considerando principalmente as metodologias USEPA, ATSDR e OMS, as quais revelaram ter a maior aplicação em artigos reportados na literatura nos últimos anos. O propósito central deste estudo foi abrir, em um marco único, as vantagens, desvantagens e as ferramentas incluídas nas metodologias reportadas. Esta análise visa, por um lado, oferecer subsídios para a possível elaboração de padrões de novas metodologias de avaliação de riscos à saúde e meio ambiente; e por outro lado, disponibilizar suporte informativo para a seleção das metodologias mais adequadas a serem aplicadas em cada caso. São discutidas as áreas cobertas por cada metodologia, suas diferenças e procedimentos comuns, assim como questões do processo avaliativo de risco que ainda permanecem em aberto. A análise realizada na presente pesquisa poderá contribuir na possível uniformização de métodos de avaliação de risco às condições da saúde coletiva e aos ecossistemas brasileiros, e para subsidiar a formulação de uma nova metodologia mais adequada e completa.

PALAVRAS-CHAVES: Avaliação de Risco; Risco Ambiental; Risco à Saúde.

\section{INTEGRATIVE REVIEW ON THE APPLICATION OF THE METHODOLOGIES FOR EVALUATING RISKS TO HEALTH AND THE EVIRONMENT}

\section{ABSTRACT}

Is known that there isn't a uniform methodology of the risk assessment, in health and environment. In this work was performed an extensive review in national and international scientific publications that apply the main methodologies for risk assessment. Was also presented, a detailed comparative analysis of the risk assessment process, especially considering the methodologies USEPA, ATSDR and WHO. In the last couple of years these methods showed to have the greatest application in reported research. The purpose of this study was to open a discussion about the advantages and disadvantages and the tools included in the reported methods. This analysis aims, firstly to offer subsidies for the possible development of new patterns of risk assessment methodologies, and secondly to provide enough information that will help select the most appropriate methodologies to be applied in each case. Were analyzed cover areas by each methodology, their differences and common procedures, as well issues of the risk assessment process that still remain open. The analysis performed in this study may contribute to the possible standardization of risk assessment methods that will to take in consideration the public health conditions and the Brazilian ecosystems. This will also support the formulation of a new and improve methodology.

KEYWORDS: Risk Assessment; Environmental Risk; Health Risk
Revista Ibero-Americana de

Ciências Ambientais, Aquidabã, v.5, n.1, Dez 2013, Jan, Fev, Mar, Abr, Mai 2014.

ISSN 2179-6858

SECTION: Articles

TOPIC: Gestão Ambiental

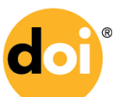

DOI: 10.6008/SPC2179-6858.2014.001.0007

Sergio Fred Ribeiro Andrade Universidade Estadual de Santa Cruz, Brasil http://lattes.cnpq.br/2756638501081370 sergiof@uesc.br

Fermin Garcia Velasco

Universidade Estadual de Santa Cruz, Brasil http://lattes.cnpq.br/2679073082540151 fermin@uesc.br

Francisco Heriberto Martínez Luzardo Universidade Estadual de Santa Cruz, Brasil http://lattes.cnpq.br/4333332503448953 fmartinezluzardo@gmail.com

Received: 28/10/2013

Approved: 15/04/2014

Reviewed anonymously in the process of blind peer

Referencing this:

ANDRADE, S. F. R.; VELASCO, F. G.; LUZARDO, F. H. M. Revisão integrativa sobre aplicação das metodologias de avaliação de riscos à saúde e ao ambiente. Revista Ibero-Americana de Ciências Ambientais, Aquidabã v.5, n.1, p.95-114, 2014. DOI: http://dx.doi.org/10.6008/SPC21796858.2014 .001 .0007 


\section{INTRODUÇÃO}

A OMS/IPCS (2010) estima que mais de $25 \%$ da incidência global de doenças está ligada a fatores ambientais, incluindo a exposição a substâncias químicas tóxicas. A exposição ao chumbo, por exemplo, é responsável por $3 \%$ da incidência de doenças cerebrovasculares e $2 \%$ de doença isquêmica do coração, no mundo. Cerca de $9 \%$ da incidência global de câncer de pulmão é atribuível à exposição ocupacional por substâncias tóxicas, e 5\% à poluição do ar.

Os problemas originados pelos contaminantes dispostos no meio ambiente estão fundamentalmente ligados a atividade humana. As atividades econômica, industrial e agrícola são as principais fontes que provocam a poluição ambiental. A análise de avaliação de risco se revela como uma ferramenta importante na busca de soluções a estes problemas. Em trabalho recente de Souza (2012), é destacada a atualidade e importância do tema, e, em particular, a potencialidade da utilização desta ferramenta para auxiliar os Sistemas de Gestão Ambiental do setor produtivo.

Desta forma, os diferentes modelos de avaliação de risco precisam incluir recursos e métodos que permitam aferir, de forma clara e objetiva, os riscos reais, com instrumentos para mensuração dos perigos e medidas para mitigação das ações nocivas como auxílio à tomada de decisões.

Nos Estados Unidos, União Europeia e em outros países, os procedimentos de avaliação de risco à saúde humana por produtos nocivos fazem parte de uma legislação influente que inclui a aplicação de procedimentos bem definidos para eliminação das fontes emissoras de resíduos perigosos. Mesmo assim, ainda são metodologias sem unificação, com abordagens diversas e muitas vezes são direcionadas para avaliar riscos no processo industrial.

Outra questão importante diz respeito à "importação" de metodologias, e até de bases de dados com referências toxicológicas, provenientes dos Estados Unidos e da Europa, por exemplo, para aplicação, por países em desenvolvimento, sem as necessárias adequações nos parâmetros regionais ou locais.

Segundo o Ministério da Saúde (2006), no Brasil, os métodos utilizados para esses fins são aplicados com base na metodologia da ATSDR (Agency for Toxic Substances and Disease Registry), o que não necessariamente considera a realidade situacional, ambiental e regional brasileira.

Nesse contexto, surgiu a motivação para o presente trabalho de revisão, em consideração à ausência de uma linguagem comum para tratamento de avaliação de riscos ambientais e à saúde, assim como da necessidade de comparação dos diversos procedimentos, nos mais variados métodos de avaliação, com propósito de contribuir para uma futura indicação de métodos para padronização de metodologias aplicadas à avaliação e gestão do risco à saúde e aos ecossistemas brasileiros. 
Nas atividades regulares as empresas produzem, manipulam, armazenam e transportam muitas substâncias tóxicas, decorrentes das especificidades das operações industriais, petrolíferas, químicas, petroquímicas, gasodutos, mineração, frigoríficas, entre outras. Por estas razões, em uma única indústria coexistem inúmeras fontes de riscos.

Segundo Lima (2009), um único enfoque ou procedimento geral não é recomendado em processo de avaliação de risco por substâncias nocivas. A autora defende que a aplicação dos métodos deve considerar especificação e avaliação da exposição e avaliação da dose e efeito como resposta à exposição, para ser teoricamente válida. Em USEPA (1989) é proposto que os métodos produzam, em geral, cálculos para exposição de risco, que expressem a realidade e que considerem as substâncias químicas, suas especificações e os possíveis efeitos adversos à saúde humana e ao meio ambiente.

Outros autores, como Demidova e Cherp (2005), apontam que em geral, os resultados das análises de avaliação de riscos são utilizados para a execução de medidas de mitigação que têm o objetivo de prevenir ou reduzir os impactos associados aos projetos; assim como restaurar ou compensar o prejuízo causado para o meio ambiente e danos à saúde humana, consistindo o processo nas seguintes etapas: 1) identificar ações de medidas de mitigação apropriadas; 2) avaliar a sua eficácia, a viabilidade tecnológica e econômica e aceitabilidade do ponto de vista público em geral; e, 3) estimar o impacto residual após a implementação dessas medidas.

Baseado nessas premissas, este estudo objetivou identificar elementos comuns e diferenças das metodologias mais utilizadas de avaliação de risco à saúde e meio ambiente, e levantar as questões em aberto, presentes nestas.

\section{METODOLOGIA}

Foram consultados, além de artigos científicos publicados em periódicos especializados, publicações da CETESB (Companhia Ambiental do Estado de São Paulo), base de dados da BIREME (Centro Latinoamericano e do Caribe de Informação em Ciências da Saúde), da base de dados científicos da SciVerse Science Directc/Elsevier, base de dados do Science Inventory, da USEPA (Environmental Protection Agency), base de dados da ATSDR (Agency for Toxic Substances and Disease Registry), base de dados da Agência Europeia para Segurança e Saúde no Trabalho, e biblioteca da OMS (Organização Mundial da Saúde).

As produções científicas foram coletadas durante o primeiro trimestre de 2013 , utilizandose palavras-chaves como metodologia de riscos, avaliação de risco, risco à saúde, risco ao meio ambiente, métodos para avaliação de riscos, framework de avaliação de risco, e riscos na segurança. Foi escolhido o período de publicações das produções científicas do ano de 2000 até 2013, num total de 197 trabalhos entre artigos, revisões, teses e dissertações.

As coletas das produções científicas foram realizadas dentro da temática da metodologia de avaliação de risco à saúde e ao meio ambiente, na abordagem mais ampla possível, 
considerando os tópicos: 1) avaliação de risco à saúde 2) avaliação dos riscos ecológicos; e 3) análise de risco na segurança em processos e instalações.

Foi reportado, no trabalho de Tixer et al. (2002), que existem mais de 60 métodos para avaliação de risco à saúde e ao meio ambiente, os quais contemplam três principais etapas analíticas: a) fase de identificação - especificação das atividades perigosas, produtos e equipamentos; b) fase de avaliação da exposição e toxicidade, através da abordagem determinística e/ou probabilística; e c) fase da caracterização de risco, que hierarquiza as margens de riscos em grau de comprometimento e perigo à saúde ou ao meio ambiente.

Dentre os trabalhos consultados, foram considerados os que tinham similaridade com a abordagem da avaliação de risco e contemplavam as fases da caracterização do perigo, avaliação da exposição nos diversos compartimentos ambientais, avaliação da toxicidade e caracterização da margem de risco. Outro critério prioritário para selecionar as metodologias em foco neste trabalho foi o grau de aceitação desses métodos, medido pela citação em outros trabalhos, utilização e aplicação em estudos concretos publicados.

Em razão de algumas limitações apresentadas, uma parte dos trabalhos consultados foi desconsiderada neste estudo, em razão de não apresentarem caracterização da margem de risco ou da aplicação de suas etapas terem gerado apenas resultados parciais. As limitações apresentadas foram: a) metodologia muito generalista que pouco considerava a especificidade do caso estudado; b) metodologia muito específica e que não considerava outras situações para avaliação, como diversidade nos compartimentos ambientais ou grupos sensíveis na população envolvida; c) poucas especificações, conceituações e proposições sugeridas; d) pouca atualização dos dados registrados na base considerada; e) falta de guia ou procedimentos específicos para situações avaliativas; e f) falta de integração com outras metodologias de avaliação, tornando-se inflexível. Os trabalhos que não tinham relação como objeto ou objetivo da pesquisa, ou limitações nos métodos aplicados, foram descartados.

Entre as diversas metodologias adotadas mundialmente, foram escolhidas as mais utilizadas pelos especialistas e as mais citadas nas publicações mundiais. A Tabela 1 apresenta as metodologias de avaliação de risco que foram consideradas referenciais na revisão bibliográfica.

Tabela 1: Metodologias consideradas para revisão.

\begin{tabular}{|l|l|}
\hline \multicolumn{1}{|c|}{ METODOLOGIAS CONSIDERADAS } & \multicolumn{1}{c|}{ REFERÊNCIA } \\
\hline Análise de Causas e Consequências & Nicolet-Monnier (1996) \\
\hline Análise Histórica de Acidentes & Khan e Abbasi (1998a) \\
\hline Ferramenta de Avaliação de Riscos & Oien et al. (1998) \\
\hline Inspeção de Segurança & Chung e Yang (1998) \\
\hline Lista de Verificação de Riscos & Khan e Abbasi (1998b) \\
\hline Metodologia de Riscos e Operabilidade - HAZOP & Kennedy e Kirwan(1998); Rogers (2000) \\
\hline Metodologia Análise Preliminar de Perigo - APP & CCPS (1989) \\
\hline Metodologia Falhas Modelos e Efeitos - FEMEA & Vanderbrande (1998) \\
\hline Metodologia Falhas em Árvores - FTA & Vesely et. al. (1981); IEC (1990) \\
\hline Metodologia Falhas por Eventos - ETA & ETA (1985) \\
\hline Metodologia da CETESB-SP & CETESB (2003) \\
\hline Metodologia da USEPA & USEPA (1989) \\
\hline
\end{tabular}




\begin{tabular}{|l|l|}
\hline Metodologia da ATSDR & ATSDR (2005) \\
\hline Metodologia da União Europeia & UE-IHCP ( 2003) \\
\hline Metodologia da OMS & OMS/IPCS (2010) \\
\hline Método somente por Dose-Resposta & NCR (1983); USEPA (1989); OMS (2010) \\
\hline Outras Metodologias & $\begin{array}{l}\text { Troutt e Elsaid (1996); Dolladille (1999); Kennedy \& Kirwan } \\
(1998) ; \text { Toola (1992) Pitblado, Williams e Slater (1990) }\end{array}$ \\
\hline
\end{tabular}

Após a seleção das publicações, estas foram classificadas de acordo com a metodologia aplicada em cada trabalho. Do resultado desta classificação foi implementado um banco de dados com os seguintes atributos: título, ano de publicação, periódico de publicação, autores, país de origem, palavras-chaves, resumo do trabalho, métodos utilizados e abordagem avaliativa.

Foram selecionadas 103 produções, constantes da Tabela 2, ordenadas por metodologia e ano de publicação. As metodologias estão mostradas como USEPA, ATSDR, OMS, UE (União Europeia) e Outras, as quais são representadas pelos outros métodos menos aplicados nos trabalhos selecionados neste estudo.

Tabela 2: Trabalhos considerados na revisão integrativa.

\begin{tabular}{|c|c|c|c|}
\hline $\mathbf{N}^{\mathbf{0}}$ & BIBLIOGRAFIA & ANO & MÉTODO \\
\hline 1 & $\begin{array}{l}\text { ROBERT L. SIELKEN JR., et. al. Regulatory Toxicology and Pharmacology, volume 65, 2, 2013, p. 214- } \\
225 .\end{array}$ & 2013 & USEPA \\
\hline 2 & $\begin{array}{c}\text { BOLGER, MICHAEL, et. al. Regulatory Toxicology and Pharmacology, volume 66, Issue 2, July } \\
2013 \text {, Pages } 234-240 .\end{array}$ & 2013 & USEPA \\
\hline 3 & MUSHAK, Science of the Total Environment 443, 2013: 643-649. & 2013 & USEPA \\
\hline 4 & AMJAD, HIRA, et. al. Ecotoxicology and environmental safety, volume 91, 1 May 2013, Pages 25-31 & 2013 & USEPA \\
\hline 5 & RODRIGUES, SARAH K., et. al. Marine environmental research, XXX, 2013, 1 - 12. & 2013 & USEPA \\
\hline 6 & HOUETO, PAUL, et. al. Regulatory Toxicology and Pharmacology 62.1, 2012: 41-48. & 2012 & USEPA \\
\hline 7 & SIIRILA, ERICA R., AND REED M. MAXWELL. Science of the Total Environment 431, 2012: 221-232. & 2012 & USEPA \\
\hline 8 & NIEMEYER, JÚLIA CARINA, et al. Applied Soil Ecology 59, 2012: 96-105. & 2012 & USEPA \\
\hline 9 & XU, LINYU, AND XIN SHU. Procedia Environmental Sciences 13, 2012: 288-296. & 2012 & USEPA \\
\hline 10 & XIE, JIANPING, et. al. Regulatory Toxicology and Pharmacology 62.2, 2012: 355-362. & 2012 & USEPA \\
\hline 11 & YEGANEH, MOJGAN, et. al. Journal of hazardous materials, 2012. Volumes $244-245,15$, p. $225-239$ & 2012 & USEPA \\
\hline 12 & COLMAN LERNER, J. E., et. al. Atmospheric Environment 55, & 2012 & USEPA \\
\hline 13 & $\begin{array}{l}\text { CHEN, MING-JEN, et. al. Food and Chemical Toxicology, 2012, volume 50, Issue 10, 2012, Pages 3867- } \\
3876\end{array}$ & 2012 & USEPA \\
\hline 14 & PERRODIN, YVES, et. al. Science of the Total Environment 431, 2012: 375-384. & 2012 & USEPA \\
\hline 15 & PERRODIN, YVES, et. al. Chemosphere, 2012, volume 90, Issu & 2012 & USEPA \\
\hline 16 & $\begin{array}{l}\text { ARNOLD, SCOTT M., et al. Regulatory Toxicology and Pharmacology, 2012, volume 64, Issue 3, p. 504- } \\
515\end{array}$ & 2012 & USEPA \\
\hline 17 & $\begin{array}{l}\text { DORNE, J. L. C. M., AND J. FINK-GREMMELS. Toxicology and Applied Pharmacology, volume 270, Issue } \\
\text { 3, } 1 \text { August 2012, Pages 187-195 }\end{array}$ & 2012 & USEPA \\
\hline 18 & $\begin{array}{c}\text { KUMAR, ARUN, AND IRENE XAGORARAKI. Regulatory toxicology and pharmacology 57.2, 2010: 146- } \\
156 .\end{array}$ & 2010 & USEPA \\
\hline 19 & $\begin{array}{r}\text { DOS SANTOS, FÁBIO NEVES, AND PEDRO AFONS } \\
2010: 139\end{array}$ & 2010 & USEPA \\
\hline 20 & DURMUSOGLU, ERTAN, et. al. Journal of hazardous materials 176.1, 2010: 870-877. & 2010 & USEPA \\
\hline 21 & SCHECKEL, KIRK G., and ROBERT G. FORD. Development & 2010 & USEPA \\
\hline 22 & WILSON, BRENDA J., et al. Annals of & 2009 & USEPA \\
\hline 23 & MERTZ, LILIANE MARCIA, et. al. & 2009 & USEPA \\
\hline 24 & HUNG, MING-LUNG, et. al. Journal of haz & 2009 & USEPA \\
\hline 25 & SILVA, ANA LICKS ALMEIDA, et. al. Ciência \& Saúde Coletiva, Rio de Janeiro 14.6, 2009: 2153-2162. & 2009 & USEPA \\
\hline 26 & $\begin{array}{c}\text { DE LIMA, CRISTIANE ANDRADE. Dissertação, Universidade Federal do Rio de Janeiro, 2009, UFRJ, Rio } \\
\text { de Janeiro. }\end{array}$ & 2009 & USEPA \\
\hline 27 & WIJNHOVEN, SUSAN WP, et al. N & 2009 & USEPA \\
\hline 28 & BOXALL, ALISTAIR BA, et al. Environmental & 2009 & USEPA \\
\hline 29 & $\begin{array}{l}\text { [88] STEINBERG, LAURA J., HATICE SENGUL, AND ANA MARIA CRUZ. Natural Hazards 46.2, 2008: } \\
143-152 .\end{array}$ & 2008 & USEPA \\
\hline 30 & CARLON, CLAUDIO, et al. Environment international 34.3, 2008: 397-411. & 2008 & USEPA \\
\hline 31 & $\begin{array}{l}\text { HAMIDIN, NASRUL, QIMING JIMMY YU, AND DES W. CONNELL. Water research 42.13, 2008: 3263- } \\
3274 .\end{array}$ & 2008 & USEPA \\
\hline 32 & SCHOENY, RITA. Human \& Ecological Risk Assessr & 2007 & USEPA \\
\hline 33 & PREUSS, PETER W., et. al. Human \& Ecological Risk Assessment, volume 13,1; (2007): 41-45. & 2007 & USEPA \\
\hline 34 & PAREKH, AMI A., and KERRY L. Human \& Ecological Risk Assessment, volume 13. (2007): 96-100. & 2007 & USEPA \\
\hline 35 & HENDERSON, ROGENE F., et al. Human \& Ecological Risk Assessment, volume 13, 1, (2007): 39-39. & 2007 & USEPA \\
\hline 36 & DERRY, CHRIS, ROGER ATTWATER, AND SANDY BOOTH. International journal of hygiene and & 2006 & USEPA \\
\hline
\end{tabular}




\begin{tabular}{|c|c|c|c|}
\hline & environmental health 209.2, 2006: 159-171. & & \\
\hline 37 & REAL, JOSÉ LUIZ GERLACH. Dissertação. Universidade Federal do Rio de Janeiro, 2005. & 2005 & USEPA \\
\hline 38 & DEMIDOVA, OLGA, AND ALEG CHERP. Environmental Impact Assessment Review 25.4, 2005: 411-429. & 2005 & USEPA \\
\hline 39 & PERES, FREDERICO, et. al. Caderno Saúde Pública 21.6, 2005: 1836-1844. & 2005 & USEPA \\
\hline 40 & PERES, FREDERICO, et al. Ciência Saúde Coletiva 10.Supl, 2005. & 2005 & USEPA \\
\hline 41 & CALDAS, E. D., et al. Food and chemical toxicology 42.11, 2004: 1877-1883. & 2004 & USEPA \\
\hline 42 & JOHNSON, B. L. Human \& Ecological Risk Assessment, volume 10, 1, (2004): 961-964. & 2004 & USEPA \\
\hline 43 & MULLER, E., et al. Atmospheric Environment 37.15, 2003: 2015-2022. & 2003 & USEPA \\
\hline 44 & HAYS, SEAN M., and LESA L. YLWARD. Regulatory Toxicology and Pharmacology 37.2 (2003): 202-217. & 2003 & USEPA \\
\hline 45 & FILIZOLA, HELOISA FERREIRA, et al. Pesquisa Agropecuária Brasileira 37.5, 2002: 659-667. & 2002 & USEPA \\
\hline 46 & WCISLO, ELEONORA, et al. Chemosphere 47.5, 2002: 507-515. & 2002 & USEPA \\
\hline 47 & FUERTAW, EDWIN J. Toxicology and industrial health 17.5-10 (2001): 302-314. & 2001 & USEPA \\
\hline 48 & STEINEMANN, ANNE. Environmental Impact Assessment Review 20.6, 2000: 627-645. & 2000 & USEPA \\
\hline 49 & CALDAS, ELOÍSA DUTRA, AND L. C. K. R. SOUZA. Revista de Saúde Pública 34.5, 2000: 529-37. & 2000 & USEPA \\
\hline 50 & $\begin{array}{l}\text { WANG, CHEN-CHEN, et. al. Journal of Hazardous Materials, volume 262, } 15 \text { November 2013, Pages 179- } \\
188\end{array}$ & 2013 & ATSDR \\
\hline 51 & $\begin{array}{l}\text { C. ROUSSELLE, J.N. ORMSBY, et. al. Regulatory Toxicology and Pharmacology, volume 65-1, 2013, p.7- } \\
11 .\end{array}$ & 2013 & ATSDR \\
\hline 52 & JAMIN, PIERRE, et. al. Journal of contaminant hydrology $127.1,2012$, p. 65-75. & 2012 & ATSDR \\
\hline 53 & ELLIS, J. BRYAN, et. al. Science of the Total Environment 416, 2012: 172-179. & 2012 & ATSDR \\
\hline 54 & KORN, MARIA GRAÇAS A., et. al. Microchemical Journal 96.1, 2010: 12-16. & 2010 & ATSDR \\
\hline 55 & NIEMEYER, JÚLIA C., et. al. Journal of Soils and Sediments 10.8, 2010: 1557-1571. & 2010 & ATSDR \\
\hline 56 & MA, HWONG-WEN, et. al. Environment international 33.2, 2007: 206-218. & 2007 & ATSDR \\
\hline 57 & HARRISON, PAUL TC, et. al. Issues in Environmental Science and Technology 22.22, 2006, p. 65-83. & 2006 & ATSDR \\
\hline 58 & $\begin{array}{l}\text { KOO, HYUN JUNG, et. al. Journal of Toxicology and Environmental Health, Part A 67.23-24, 2004, p. 1901- } \\
1914 .\end{array}$ & 2004 & ATSDR \\
\hline 59 & XELEGATI, ROSICLERE. et. al. Revista Latinoamericana de enfermagem, 11.3, 2003, p. 350-356. & 2003 & ATSDR \\
\hline 60 & PATTERSON, JACQUELINE, et. al. Toxicol & 2002 & ATSDR \\
\hline 61 & $\begin{array}{c}\text { SPENGLER, ROBERT F., et. al. International journal of hygiene and environmental health } 205.1,2002, p . \\
77-83 .\end{array}$ & 2002 & ATSDR \\
\hline 62 & FERON, V. J., and J. P. GROTEN. Food and chemical toxicology 40.6 , 2002, p. 825-839. & 2002 & ATSDR \\
\hline 63 & MAISTRO, LILIANE CORREA. Revista. Nutrição 14.3, 2001, p. 219-224. & 2001 & ATSDR \\
\hline 64 & SANTOS, LUIS FERNANDES PEREIRA, et. al. Food Control, & 2013 & OMS \\
\hline 65 & POHJOLA, M. V., et. al. Food and Chemical Toxicolo & 2012 & OMS \\
\hline 66 & $\begin{array}{l}\begin{array}{l}\text { RECKMANN, K., I. TRAULSEN, AND J. KRIETER. Journal of environmental management 107, 2012: 102- } \\
109 .\end{array} \\
\end{array}$ & 2012 & OMS \\
\hline 67 & $\begin{array}{l}\text { MARANO, KRISTIN M., et. al. Regulatory Toxicology and Pharmacology, volume 64, Issue 2, novembro } \\
2012 \text {, Pages } 243-252\end{array}$ & 2012 & OMS \\
\hline 68 & GUILLÉN, D., et. al. Science of the Total Enviror & 2012 & OMS \\
\hline 69 & ROVIRA, JOAQUIM, et. al. Archives of environmental contam & 2011 & OMS \\
\hline 70 & TOPUZ, E., I. TALINLI, AND E. AYDIN. Environment international 37.2, 2011: 393-403. & 2011 & OMS \\
\hline 71 & NAVARRO, MARCUS VINÍCIUS TEIXEIRA, et. al. Ciência \& Saúde Coletiva 2, 2010, p. 3477-3486. & 2010 & OMS \\
\hline 72 & NETO, MARIA DE LOURDES FERNANDES, et. al. Engenharia Sanitária Ambiental 14.1, 2009: 69-78. & 2009 & OMS \\
\hline 73 & PENNINGS, E. J. M., et. al. Regulator & 2008 & OMS \\
\hline 74 & SOLECKI, ROLAND, et. al. Food and Che & 2005 & OMS \\
\hline 75 & VAN DEVENTER, T. E., et. al. Progress in biophy & 2005 & OMS \\
\hline 76 & $\begin{array}{l}\text { SHARIFF AZMI MOHD, AND DZULKARNAIN ZAINI. Journal of Loss Prevention in the Process Industries, } \\
\text { volume 26, Issue 4, July 2013, p. 605-613. }\end{array}$ & 2013 & UE \\
\hline 77 & WOLLENWEBER, JENS, et. al. Energy Procedia 37, 2013: 4825-4832. & 2013 & UE \\
\hline 78 & SWARTJES, F. A., et. & 2012 & UE \\
\hline 79 & ASCHBERGER, KARIN, et. al. Environm & 2011 & UE \\
\hline 80 & MUÑOZ, IVAN, $\epsilon$ & 2010 & UE \\
\hline 81 & NIEUWENHUIJSEN, et. al. Environment international 32.8, 2006: 996-1009. & 2006 & UE \\
\hline 82 & FRYER, MICHAEL, ET AL. Environmental Science \& Policy 9.3, 2006: 261-274. & 2006 & UE \\
\hline 83 & OLSEN, STIG IRVING, ET AL. Environmental Impact Assessment Review 21.4, 2001: 385-404. & 2001 & UE \\
\hline 84 & VIANCO, MARTA G. el al. Atmospheric Environment, Volume 40, Issue 7, March 2006, Pages 1189-1198. & 2009 & CETESB \\
\hline 85 & $\begin{array}{c}\text { COELHO, SUANI TEIXEIRA, et. al. Energy for Sustainable Development, volume 10, Issue 2, June 2006, } \\
\text { Pages 26-39. }\end{array}$ & 2008 & CETESB \\
\hline 86 & $\begin{array}{c}\text { FREITAS, CLARICE UMBELINO DE, et. al. Environmental Research, volume 103, Issue 3, 2007, p 338- } \\
344 .\end{array}$ & 2006 & CETESB \\
\hline 87 & GÜNTHER, WANDA M. RISSO. São Paulo em Perspectiva 20.2, 2006: 105-117. & 2006 & CETESB \\
\hline 88 & $\begin{array}{l}\text { GIULIANO, ANTONIO DONIZETTI, ET. AL. XXVIII Congresso Interamericano de Ingeniería Sanitaria y } \\
\text { Ambiental, Gestión inteligente de los recursos naturales: desarrollo y salud. México, 2002, p } 1 \text { - } 7\end{array}$ & 2002 & CETESB \\
\hline 89 & $\begin{array}{c}\text { BROLLO, MARIA JOSÉ. Congresso Brasileiro de Engenharia Sanitária e Ambiental, } 21 \text { 4. ABES, 2001. p. } \\
\text { 1-24. }\end{array}$ & 2001 & CETESB \\
\hline 90 & $\begin{array}{l}\text { PASMAN, HANS, AND GENSERIK RENIERS. Journal of Loss Prevention in the Process Industries, } 2013 . \\
\text { p. 1-8. }\end{array}$ & 2013 & Outro \\
\hline 91 & SIIRILA, ERICA R., et. al. Advances in Water Resources 36, 2012: 146-164. & 2012 & Outro \\
\hline 92 & $\begin{array}{l}\text { MCKNIGHT, URSULA S., AND MICHAEL FINKEL. Environmental Modelling \& Software, Volume } \\
\text { 40, February 2013, Pages 35-50. }\end{array}$ & 2012 & Outro \\
\hline 93 & AISSANI, LYNDA, et. al. Process Safety and Environmental Protection 90.4, 2012: 295-303. & 2012 & Outro \\
\hline 94 & PIZZOL, MASSIMO, et al. Journal of Cleaner Production 19.6, 2011: 646-656. & 2011 & Outro \\
\hline 95 & KOIVISTO, RAIJA, et. al. Technological Forecasting and Social Change 76.9, 2009: 1163-1176. & 2009 & Outro \\
\hline 96 & KOMLJENOVIC, et.al. Safety Science $46.5,2008:$ 792-801. & 2008 & Outro \\
\hline 97 & JESUS, KATIA REGINA EVARISTO DE, et. al. Applied Biosafety 11.3, 2006: 127. & 2006 & Outro \\
\hline
\end{tabular}




\begin{tabular}{|c|c|c|c|}
\hline $\mathbf{9 8}$ & SIMÕES FILHO, SALVADOR. Dissertação, Universidade Federal do Rio de Janeiro, 2006. & 2006 & Outro \\
\hline $\mathbf{9 9}$ & MENDES, PATRICIA B. M. TEIXEIRA. Dissertação, Universidade de São Paulo. Depart. de Saúde & 2006 & Outro \\
\hline $\mathbf{A m b i e n t a l , ~ 2 0 0 6 . ~}$ & PESSOA, MARIA CONCEIÇÃO PERES YOUNG, et. al. Pesticidas 13, 2003: 111-122. & 2003 & Outro \\
\hline $\mathbf{1 0 1}$ & VOLNEY DE MAGALHÃES CÂMARA. Revista Brasileira de Epidemiologia 6.2, 2003. & 2003 & Outro \\
\hline $\mathbf{1 0 2}$ & TIXIER, JEROME, et. al. Journal of Loss Prevention in the process industries 15.4, 2002: 291-303. & 2002 & Outro \\
\hline 103 & $\begin{array}{c}\text { BRAUTBAR, NACHMAN et. al. International journal of hygiene and environmental health 205.6 (2002): 479- } \\
491 .\end{array}$ & 2002 & Outro \\
\hline
\end{tabular}

Da classificação dos artigos selecionados, 54 produções, ou $52,4 \%$, foram publicadas entre os anos de 2009 e 2013 (até o primeiro trimestre). Acredita-se que essa situação seja ocasionada em razão da possível aplicação das tecnologias recentes disponíveis para o processo produtivo e métodos avaliativos de risco. Seguida de 20 produções, ou 19,4\%, entre 2006 e 2008, $14,6 \%$ entre 2003 e 2005 , e 13,6\% entre 2000 e 2002.

A Figura 1 mostra esses valores absolutos das publicações selecionadas por ano de publicação.

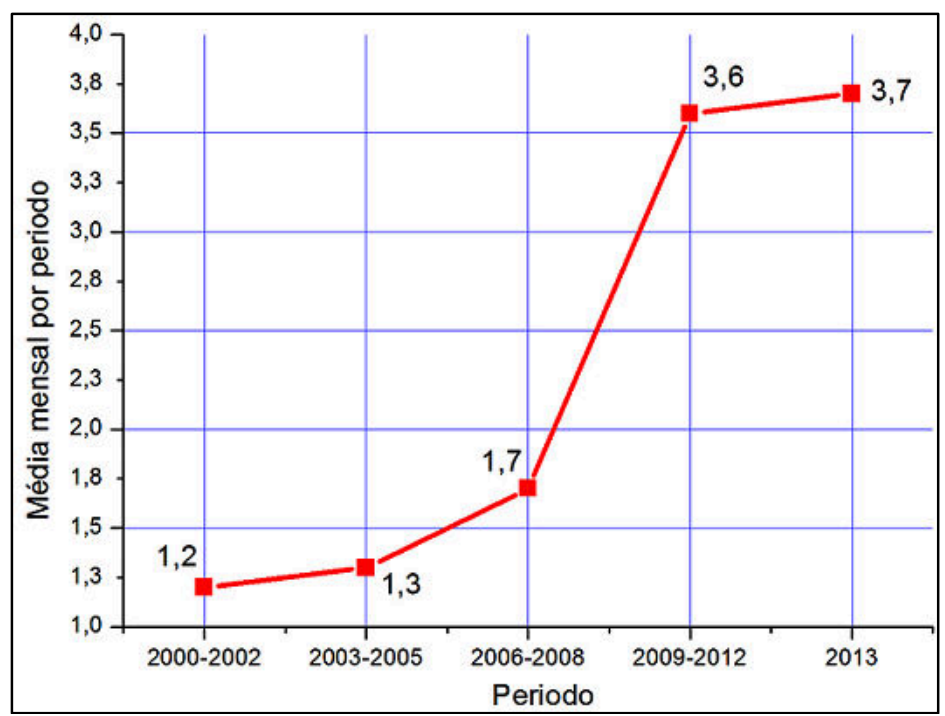

Figura 1: Média mensal de publicações por período.

Diante disso, e face o grande volume de trabalhos selecionados, foi realizada uma categorização conforme local ou País de publicação para conhecimento da origem das metodologias. Com isso, pôde-se elaborar uma tabela que serviu para análise da importância do tema na comunidade científica e do grau de interesse que os especialistas, governos e indústria têm na utilização de determinadas metodologias.

A Figura 2 mostra a distribuição dos artigos, revisões, teses e dissertações por País de origem de produção e publicação. Os Estados Unidos da América (EUA), em destaque, somam a quantidade de 46 trabalhos coletados com 44,7\%, seguidos por países da União Européia, com 21 trabalhos e $20,4 \%$. No Brasil, foram encontradas 20 produções publicadas, com a temática principal deste trabalho na proporção de $19,4 \%$ do total. A busca sobre a literatura no Brasil foi intensificada, apoiando-se também em teses e dissertações da área, chegando-se, por estas razões, à quantidade significativa de trabalhos comparando-se com outros Países. Essa situação 
foi justificada pelos objetivos deste trabalho que se vinculam à indicação de uma metodologia adequada às condições brasileiras.

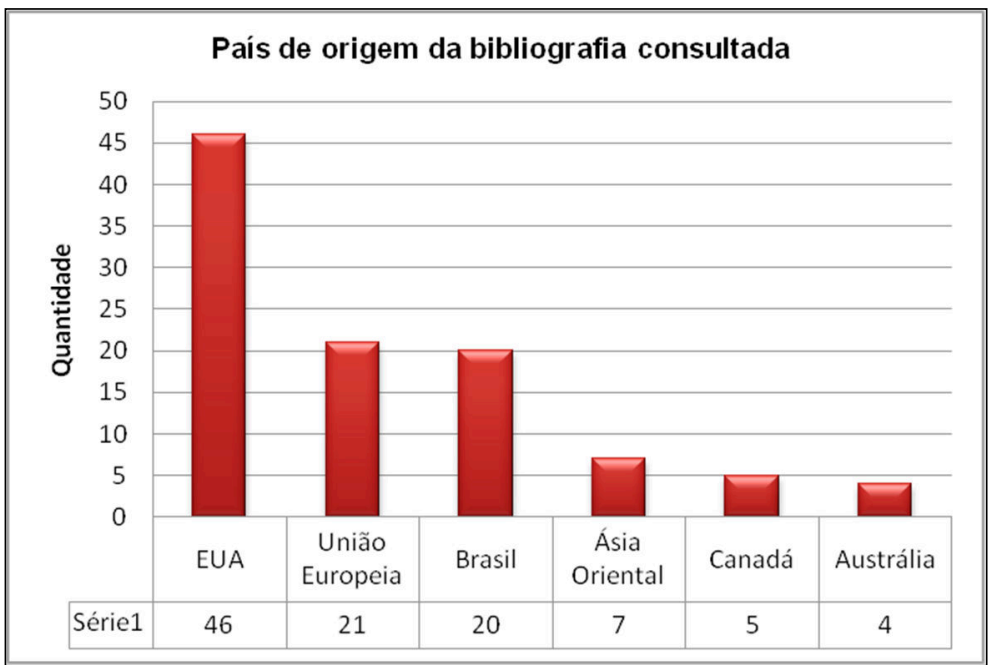

Figura 2: Trabalhos consultados por País de origem.

Em Brilhante e Caldas (2002), é citado que o rápido desenvolvimento tecnológico e industrial dos EUA fez surgir inúmeras situações de riscos em vários sítios contaminados, com forte ligação entre os produtos químicos e o surgimento de determinadas doenças, a exemplo do câncer.

Esses acontecimentos levaram o poder público a procurar medidas para gestão e mitigação dos riscos, e a comunidade científica foi estimulada a realizar pesquisas nessa área, incluindo a definição de metodologias apropriadas para o conhecimento e análise dos riscos à saúde e ao meio ambiente.

No Brasil, após a década de 1990, o governo e os especialistas têm realizado esforços para o conhecimento, estudos e pesquisas sobre avaliação de riscos, embora ainda não sejam suficientes o bastante para a sua boa gestão. Esta situação contribuiu para números significativos de trabalhos publicados que foram ilustrados anteriormente.

\section{Aspectos Gerais das Metodologias de Avaliação de Risco}

Dos artigos selecionados, foi realizada uma categorização por modalidade do método, onde foram indicadas as seguintes linhas: qualitativo, quantitativo e quali-quantitativo, com abordagens determinísticas e probabilísticas, de acordo com Tixer et al. (2002).

Segundo CETESB (2003), o método quantitativo é habitualmente empregado em análises de riscos com estimativa de frequências e consequências do perigo, aplicação de modelos matemáticos passíveis de validações, informações da avaliação dose-resposta e investigações epidemiológicas.

O método qualitativo emprega tópicos elaborados com observações e classificações contextualizadas segundo níveis comparativos em avaliação de risco, considerando as fases 
influentes do estudo, tais como avaliação da exposição dos poluentes e suas toxicidades, e os resultados dos modelos matemáticos.

Um exemplo desse tipo de método que caracteriza níveis conceituais de risco é o CatReg - Categorical Regression, cujo método inclui a regressão categórica ordinal e resulta na margem conceitual de risco que está especificado em USEPA (2006). Nesse método, deve-se indicar conceitualmente a hierarquização das margens de risco onde os mais altos níveis incluem elevadas exposições a produtos tóxicos e alta toxicidade provocando efeitos adversos à saúde até a situação letal. E, nos níveis mais baixos de risco, numa situação de menor grau de exposição e baixa toxicidade, indicam moderados efeitos adversos à saúde, podendo-se até permitir remediação, segundo Brilhante e Caldas (2002).

A Conjunção dos métodos duplos quali-quantitativo aplica as duas abordagens numa situação analítica e avaliativa mais completa. Como aponta Tixer et al. (2002), o método determinístico considera a quantificação de produtos nocivos, equipamentos e instalações, com análises em grau e níveis de riscos. Por sua vez, o método probabilístico relata níveis de falhas, cenários e probabilidade de ocorrências e consequências de perigos e riscos.

Em geral, o método determinístico é aplicado a um cenário específico, com duração precisa da exposição de determinado poluente e conhecimento definido da população ou grupos sensíveis envolvidos, assim como os males provocados.

Já o método probabilístico tem característica estocástica com alguns parâmetros prédefinidos de cenários, tais como exposição nos compartimentos do solo, subsolo, águas subterrâneas, climas pouco sazonais e de muita pluviosidade. Nestes cenários geralmente são consideradas múltiplas substâncias contaminantes, população envolvida oscilante e longa duração de exposição. Estas condições de contorno obrigam, em muitos casos, a estimação de parâmetros, com consequente avaliação de predição e tendência. Geralmente esta situação acontece nas avaliações de risco nas atividades de mineração, exploração petrolífera e metalurgia.

A Figura 3 ilustra a classificação dos métodos citados nos trabalhos selecionados, com abordagens determinísticas e probabilísticas. Desses trabalhos, 52 correspondem a abordagem quantitativa; em segundo aparece a aplicação da abordagem quali-quantitativa, com 36 trabalhos; e a abordagem quantitativa com 15.

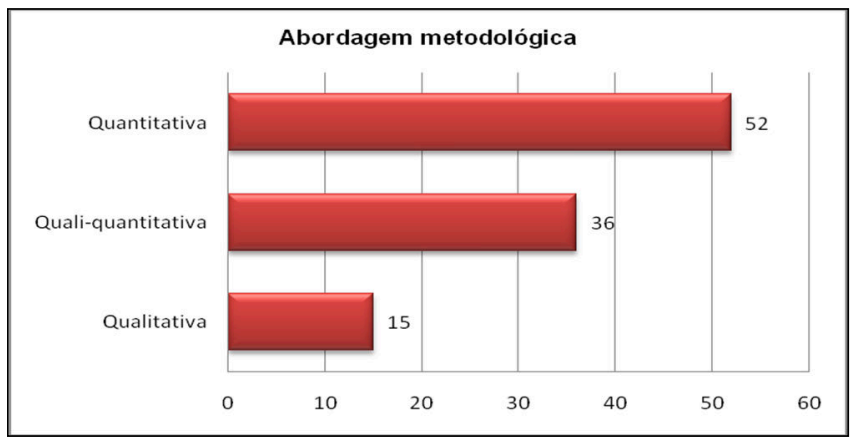

Figura 3: Aplicação de abordagem de métodos. 
Das produções científicas analisadas, segundo ilustra a Figura 4, a metodologia definida pela USEPA, em USEPA (1989), foi citada e aplicada por 49 trabalhos, correspondendo a 47,5\% do total pesquisado, seguido pela metodologia da ATSDR, em ATSDR (2005), com 14 trabalhos correspondendo a 13,6\%, e a terceira metodologia mais citada foi da OMS, em OMS (2010), com 12 trabalhos, na proporção de $11,7 \%$ das produções verificadas. A metodologia da União Européia vem aumentando a quantidade de citação em trabalhos científicos desde a publicação de sua metodologia após 2003, pela importância na área de abrangência e também pela sua exigência governamental (UE-IHCP, 2003).

Nas publicações encontradas verificamos que as outras metodologias foram aplicadas em uma escala menor de casos reportados.

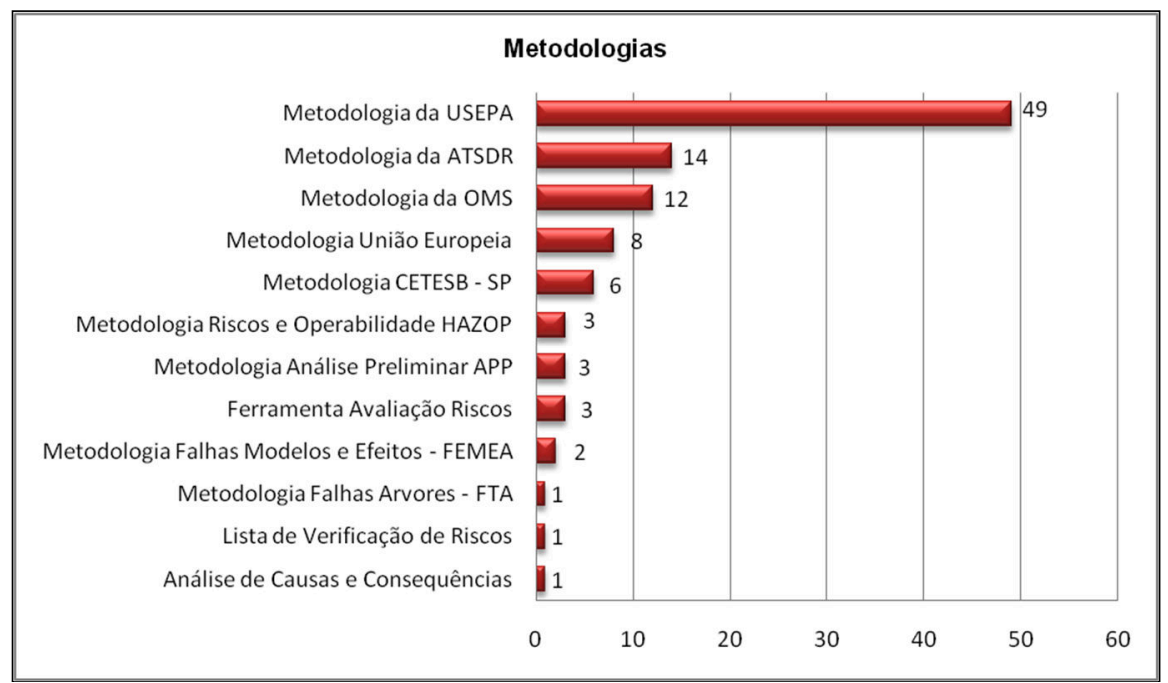

Figura 4: Metodologia por citação e aplicação nas produções.

Nas três metodologias mais aplicadas, da USEPA, ATSDR e OMS, existe um processo padrão que descreve três fases principais para uma avaliação de riscos: escopo, formulação do problema e planejamento; análise e avaliação da exposição e da toxicidade; e caracterização do risco. A modelação matemática e utilizada para a avaliação da exposição de contaminantes, a avaliação da toxicidade, e a caracterização das margens de risco. Esse roteiro foi desenvolvido em NRC (1983) é conhecido como paradigma da avaliação de risco, e tem norteado até hoje as principais metodologias para avaliação do risco à saúde e ao meio ambiente.

$\mathrm{Na}$ primeira fase, uma equipe de avaliadores especifica cenários, produtos, vias de exposição e população envolvida, estabelece as metas, amplitude, profundidade e foco da avaliação. Os resultados dessa fase servem para caracterizar a avaliação da exposição de acordo com modelos conceituais e um plano de avaliação de risco.

A fase da avaliação da exposição inclui o desenvolvimento de perfis de exposição, os compartimentos ambientais, as vias de exposições e as formas de ingestão, contatos dérmicos e inalação, considerando as interações entre os produtos tóxicos, as populações afetadas e o cenário ambiental, fauna e flora. É nessa fase que se espera serem resolvidas questões técnicas, 
tais como a toxicidade de produtos, vulnerabilidade das populações e interações entre as substâncias químicas diante das propriedades físicas do meio.

A última fase constitui a caracterização dos riscos, que busca a indicação de uma margem de risco, as especificações de risco em termos da sua importância e a confiabilidade das estimativas. É também nesta etapa que é realizada uma avaliação e verificação do processo avaliativo, conferindo se este cumpriu os objetivos e metas definidos na primeira fase.

Além destes aspectos comuns, apontados anteriormente, que as metodologias USEPA, ATSDR e OMS apresentam, é importante destacar também suas diferenças. A ênfase para aplicação dessas metodologias difere conforme a situação de risco. A ATSDR e OMS focam claramente seus esforços em ações voltadas especificamente para a saúde pública e ameaças à saúde humana. A OMS tem métodos para avaliação dos riscos nos ecossistemas, porém com moderada caracterização aos riscos inerentes e de muita complexidade de aplicação, conforme citado em OTA (1995). Por outro lado, a USEPA preocupa-se fundamentalmente em considerar tanto as atuais quanto as futuras ameaças à saúde e, sobretudo, aos impactos prejudiciais ao meio ambiente.

A metodologia da USEPA foi a mais utilizada nos trabalhos analisados para este artigo. Esta metodologia envolve atividades que se enquadram em aspectos amplos de avaliação de risco tanto para saúde humana quanto aos ecossistemas.

A política e os programas que norteiam o conjunto de técnicas que compõem essa metodologia têm origem no Fórum de Avaliação de Risco, que é uma comissão permanente formada por cientistas para discussão e deliberação sobre questões e procedimentos de avaliação. A principal abordagem dessa metodologia segue o conhecido paradigma de avaliação do risco, descrito em NRC (1983).

O conjunto de métodos da USEPA apresenta guias de práticas, procedimentos, modelos conceituais e framework's para uma ampla variedade de avaliações. São avaliações para recursos hídricos, solos nas várias camadas, ar atmosférico, diversidades de produtos químicos e suas interações, avaliação específica para exposição com metais, avaliação por microorganismos, avaliação por grupos e populações sensíveis, como também avaliações por situações adversas à saúde humana e aos impactos ambientais.

Dentre os métodos abordados pela USEPA para calcular a avaliação da exposição e caracterizar risco, utiliza-se o método da toxicologia Noael/Loael, NRC (1983), dose referência USEPA (1998), o método probabilístico BMD - Benchmark Dose, USEPA (2000), e o método CatReg - Categorical Regression, USEPA (2006).

A USEPA mantém diversos bancos de dados, a exemplo do IRIS - Integrated Risk Information System, com caracterização dos produtos químicos e seus compostos e informações sobre dose referência, nível de Noael/Loael e BMD, sintomatologia na saúde humana e estudos de bioensaios com animais. 
Porém, apesar desses recursos, seus objetivos e utilização de parâmetros estão direcionados às especificidades do território norte americano, o que presume a necessidade de ajustes para emprego em outras regiões e países.

A metodologia da ATSDR contempla um conjunto de procedimentos e informações com caracterização para efeitos de doenças e prevenção à saúde, com indicativos para análises e avaliações de riscos à saúde coletiva. Os seus objetivos são: avaliar riscos para a saúde humana em sítios contaminados com produtos tóxicos, e recomendar ações mitigadoras em prol da saúde pública; verificar a correlação entre a exposição de substâncias tóxicas e doenças; desenvolver e disponibilizar informações confiáveis e compreensíveis para as comunidades afetadas e outros interessados.

A avaliação utilizada pela metodologia da ATSDR busca identificar possíveis riscos nocivos e indicar as ações necessárias para proteger a saúde pública, sobretudo. Declinando, parcialmente, dos estudos para os riscos ambientais em razão dos seus objetivos institucionais.

Para esse fim, ATSDR considera os mesmos dados e informações da etapa inicial para avaliação de riscos, comparando-se com a metodologia da USEPA, mas concentra-se nas condições de exposição em determinados locais particulares e de interesses específicos para saúde dos grupos sensíveis afetados e da comunidade avaliada, além de dados disponíveis sobre epidemiologia para aplicar em sua avaliação. Considera também, situações e exposições precedentes de produtos nocivos, além de potenciais riscos futuros (ATSDR, 2005).

Os modelos conceituais nas duas metodologias (USEPA e ATSDR) são semelhantes (por exemplo: a coleta de dados, avaliação da exposição e a avaliação toxicológica), sempre com ênfase na avaliação para saúde pública, onde fornece perspectiva adicional integrando as condições específicas do local de exposição com os dados dos efeitos na saúde e problemas de saúde específicos da comunidade. A metodologia de avaliação da ATSDR também promove divulgação dos dados sobre os resultados da saúde quando confiáveis, para identificar as taxas de doença ou morte e se estão elevadas para determinada comunidade, especialmente se a população manifesta preocupação com algum resultado específico (por exemplo, o câncer).

O processo de avaliação de risco utilizado na metodologia da OMS começa com a formulação do problema e exige especificação detalhada da caracterização do perigo à saúde. A avaliação de risco à saúde segue o método conceitual de análise de acordo com o paradigma tradicional da NRC (1983) e faz orientação através dos vários parâmetros num roteiro de aplicação específico.

O método da OMS contém procedimentos pouco detalhados sobre parâmetros para o processo de avaliação de risco ambiental e mais detalhados para avaliação do risco à saúde humana. Indica vários modelos matemáticos para avaliação da exposição, por compartimentos, vias de exposição e populações afetadas, principalmente para grupos ocupacionais. Apresenta ferramentas adequadas para a caracterização da margem de risco, sem indicar meios para interpretações, aplicando uma abordagem mais quantitativa. O roteiro da metodologia pelo 
método da OMS respeita quatro etapas da avaliação de riscos: identificação e caracterização do perigo, a avaliação da exposição, a avaliação dos efeitos sobre dose-resposta e a caracterização da margem de risco.

A terminologia empregada pelo método da OMS está em conformidade com as definições e práticas estabelecidas pelo IPCS - International Programme on Chemical Safety, IPCS (2013), e em numerosas outras publicações. Ao longo deste documento, é frequente a indicação de valores de determinadas grandezas como referência toxicológica, a exemplo de valores de referência para ingestão diária aceitável e a ingestão diária tolerável, que servem como parâmetros para aplicação da metodologia, conforme OMS (2010).

A OMS considera dados e informações originados das seguintes entidades e bases de dados: International Chemical Safety Cards; Screening Information Datasets for High Production Volume; Chemicals WHO Recommended Classification of Pesticides by Hazard; UN Recommendations for the Transport of Dangerous Goods; IARC monographs; Hazardous Substances Data Bank; European Chemical Substances Information System; EU Classification and Labelling System International Chemical Control Toolkit.

Observa-se ainda algumas diferenças nas informações de substâncias químicas constantes das principais bases de dados toxicológicos, entre a OMS e a USEPA. A OMS utiliza a base do INCHEM - Chemical Safety Information from Intragovernmental Organizations, mantida pelo IPCS, e a USEPA utiliza o banco de dados IRIS. Ambos com dados sobre contaminantes e seus efeitos deletérios, doses referências para ingestão e concentração, pesquisas sobre carcinógenos e cancerígenos, entre outras. Essas diferenças podem levar a interpretações divergentes para situações similares.

Outras diferenças dizem respeito à aplicação de procedimentos, principalmente quanto à avaliação do meio ambiente e seus parâmetros para cálculo da margem de risco, a exemplo da PEC - Predicted Environmental Concentration e PNEC - Predicted no Effect Concentration, na metodologia da OMS, que possibilita estimar caracterização de risco tornando-o demasiadamente probabilístico. Os critérios da USEPA são baseados em parâmetros mais determinísticos e aplicados com mais rigor.

\section{RESULTADOS}

Segundo Brilhante e Caldas (2002), os problemas relativos aos contaminantes ambientais e à preservação dos ecossistemas estão associados e se refletem, sobremodo, na saúde humana. Estes autores Esses citam ainda que o processo de avaliação de risco ambiental é um instrumento metodológico influente para a execução de uma política de saúde coletiva.

Pelas características da interação entre homem e ambiente, pode-se observar que há uma agregação natural entre a avaliação dos riscos à saúde humana e ao meio ambiente. Esta situação ficou evidente, diante da expressiva quantidade de aplicação das metodologias da 
USEPA e OMS observadas neste estudo. Pois a inter-relação entre a saúde humana e o meio ambiente obriga a uma análise adequada da questão de risco e que esta seja realizada de forma integrada.

Em razão da predominante aplicação dessas três metodologias, foi elaborada uma tabela comparativa com os principais procedimentos e fatores que compõem os roteiros e etapas necessárias para o processo de avaliação. Considerou-se também a metodologia aplicada em CETESB (2003) por ser adaptável à realidade brasileira, muito embora sabendo-se que tal método inicialmente considerou somente acidentes industriais ocorridos na década de 1980, especialmente no segmento da indústria petroquímica.

A Tabela 3 mostra indicações de etapas e procedimentos para avaliação de risco à saúde humana e ao meio ambiente que foram encontrados na análise dos procedimentos metodológicos aplicados, no conjunto total de artigos revisados.

O princípio norteador para organização dos procedimentos indicados na Tabela 3 foi o paradigma de avaliação de risco proposta por NRC (1983), que referencia a caracterização do perigo, avaliação da exposição, avaliação dose-resposta e caracterização do risco como etapas básicas para avaliação e o conhecimento de riscos. Outros procedimentos foram adaptados das metodologias menos citadas, mas foram considerados importantes para o conhecimento da margem de risco, mitigação das ações e gestão dos riscos.

Foram considerados três grandes grupos de fatores importantes para o processo de avaliação, qualificados como: a) identificação e especificação de riscos; b) modelos de avaliação, avaliação da exposição e toxicidade; e c) planejamento e gestão de riscos. Em cada fator foram identificadas etapas importantes para a realização da avaliação e caracterização do risco.

$\mathrm{Na}$ Tabela 3, todos os procedimentos em cada metodologia (USEPA, ATSDR e OMS) foram comparados e registrados com a grafia de um "ponto preto", na respectiva linha quando o procedimento forma parte da metodologia e, deixado sem notação quando não está incluído, caracterizando um registro binário de dados (1-presença; 0-ausência). Cada procedimento foi adotado como amostra $\boldsymbol{n}$ e cada metodologia como grupo pertencente a $\boldsymbol{m}$ colunas, formando uma matriz de dados $\boldsymbol{D}$ composta por $\boldsymbol{n} \times \boldsymbol{m}$ elementos. Cada procedimento atendido foi registrado por 1 que representa um objeto no espaço $n$-dimensional, podendo ser agrupado com outros que sejam próximos e assemelhados a ele (NETO e MOITA, 1998).

Desta forma, as similaridades ou dissimilaridades entre as amostras foram caracterizadas como função da distância entre dois pontos no espaço n-dimensional, calculada através da distância euclidiana com abordagem de vizinhos mais próximos, utilizando o método de classificação hierárquica aplicado com uso do pacote estatístico SPSS (Statistical Package for the Social Sciences, versão 18.0.

Os gráficos do tipo dendrograma foram gerados e mostrados na Figura 5 e ilustram os resultados dos procedimentos adotados. O dendrograma A corresponde aos agrupamentos dos 
procedimentos metodológicos e o dendrograma B corresponde aos agrupamentos por metodologia, identificados na Tabela 3.

Tabela 3: Comparativo de fatores de avaliação.

\begin{tabular}{|c|c|}
\hline Ordem & Etapas/Procedimentos \\
\hline A & \\
\hline 01 & Identificação do perigo \\
\hline 02 & Especificação do risco \\
\hline 03 & $\begin{array}{l}\text { Inventariar produtos perigosos para cada } \\
\text { cenário }\end{array}$ \\
\hline 04 & $\begin{array}{l}\text { Classificar os poluentes de acordo com o } \\
\text { inventário e normas vigentes }\end{array}$ \\
\hline 05 & Identificação das falhas potenciais \\
\hline 06 & Informações de segurança de processo \\
\hline
\end{tabular}

Descrição
IDENTIFICAÇÃo E ESPECIFICAÇÃo DE RISCOS
Técnicas de identificação de perigo; Análise preliminar de
perigos; Identificação da população afetada.
perigos; Identificação da população afetada.

Especificação dos riscos, tipos de riscos, características de riscos.

Levantamento, contagem, descrição e quantificação de produtos perigosos em cada cenário reconhecido.

Classificação das substâncias, suas especificações e possíveis efeitos em conformidade com o inventário realizado.

Especificação das falhas possíveis de acontecimento para prévio conhecimento das ações adotadas.

Registro de documentos atualizados visando garantir adequada avaliação de riscos sobre substâncias químicas, tecnologia, equipamentos e processos.

\begin{tabular}{|l|l}
\hline 07 & Dividir o sítio em unidades funcionais \\
\hline 08 & Avaliação da dose-resposta
\end{tabular}

EXPOSIÇÃO E TOXICIDADE

Conhecimento da geografia das instalações e a divisão em territórios conforme exposição e concentração de produtos.

Utilização de testes laboratoriais, em animais, visando conhecimento das concentrações toleráveis à exposição de produtos nocivos e sua extrapolação para humanos.

\begin{tabular}{|l|l|}
\hline 09 & $\begin{array}{l}\text { Avaliação da exposição de produtos } \\
\text { nocivos }\end{array}$ \\
\hline 10 & $\begin{array}{l}\text { Estimativa de efeitos à população, biota e } \\
\text { avaliação de vulnerabilidade }\end{array}$ \\
\hline
\end{tabular}

11 Estimativa de avaliação de riscos à interação humana

12 Estimativa de frequência de eventos

13 Estimativa de riscos toleráveis

14 Cálculo da emissão de substâncias na ocorrência de falhas

15 Avaliação do impacto e dos efeitos adversos na saúde das pessoas e no meio ambiente

16 Interação entre os sítios contaminados por compartimento ambiental

17 Pesquisar casos de possível disposição química

18

Agrupar os casos de emissões semelhantes

19 Calcular taxas das emissões por cenários específicos

20 Agrupar as emissões por taxas

21 Previsão segundo modelos de incerteza; calcular as consequências; apresentar os resultados

22 Elaborar mapa dos efeitos versus distâncias

23 Estimar as frequências dos eventos

24 Interpretar os resultados por situações e cenários

25 Critérios para validação de modelos

26 Modelo matemático para extrapolação de resultados.

\begin{tabular}{|l|l|}
\hline C & Revisão dos riscos de processos \\
\hline 27 &
\end{tabular}

28 Gerenciamento de modificações

29 Manutenção e garantia de integridade de sistemas

30 Procedimentos operacionais e de supervisão
Determinação de modelos e suas aplicações para conhecimento da concentração de agentes pela exposição a produtos nocivos. Consideração de condições atmosféricas; condições topográficas; solos, hídricos, tempo e periodicidade de exposição; Cálculo da explosão por compartimentos e vias de absorção.

Riscos sociais; risco individual; riscos econômicos.

Probabilidade de acontecimento de perigo e riscos.

Descrição qualitativa da estimativa dos riscos e consequências toleráveis à saúde.

Modelo matemático para estimação de emissão de produtos nocivos após acontecimento de falhas em processos.

Estimação do resultado da exposição à produtos químicos, considerando avaliação dose-respostas, por período de tempo.

Interação das unidades de riscos por compartimento de ar, solo e água, visando investigação e o conhecimento de casos cumulativos de contaminantes.

Investigação de casos de falhas e possíveis ações geradoras de disposição de produto químico ao meio.

Agrupamento e associação de situações de emissões perigosas, visando melhor gerenciamento.

Modelo matemático para cálculo das emissões de contaminantes por cenário envolvido.

Agrupamento e associação das emissões por taxas toleráveis ou não à saúde.

Probabilidade das consequências e previsão de resultados de riscos; utilização de métodos Monte Carlo ou QSAR, para predição de exposição quando não houver parâmetros definidos.

Mapeamento e lay-out das distâncias das situações de riscos e seus efeitos por área geográfica.

Estimativa das frequências dos eventos por tempo e situações de riscos.

Interpretação e análise de resultados para contribuição ao processo de avaliação de riscos.

Validação dos modelos através de critérios reconhecidos e métodos aplicáveis.

Avaliação e cálculo para extrapolação de resultados da avaliação dose-resposta, de bioensaios com animais para humanos.

\section{PLANEJAMENTO E GESTÃO DE RISCOS}

Análise e verificação da implementação do projeto, desde a fase inicial de instalações e processos até as atividades operacionais. Implementação de sistema gerencial para adequações às modificações de processos para garantir segurança à saúde humana e ao meio ambiente.

Provimento de garantia e segurança sobre processamento, armazenamento, transporte de substâncias químicas.

Instruções precisas que propiciem descrição das etapas dos processos para supervisão e operações.
USEPA ATSDR OMS
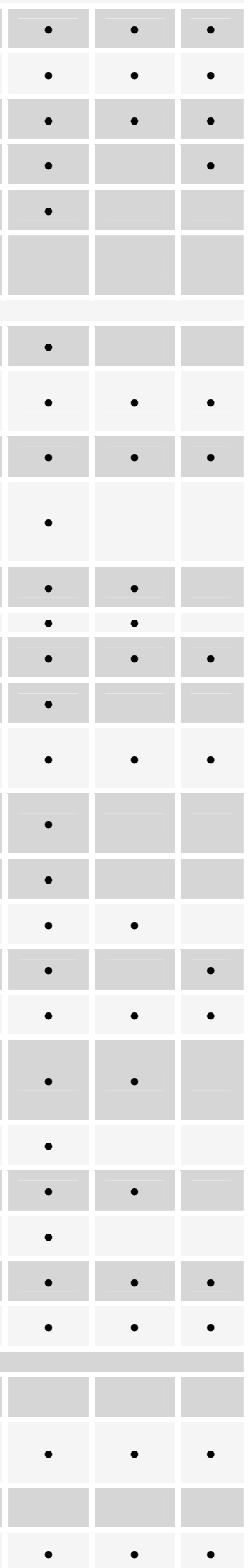


\begin{tabular}{|c|c|c|c|c|c|}
\hline 31 & Capacitação dos recursos humanos & $\begin{array}{l}\text { Treinamento de sistemas, processos e tecnologias para garantir } \\
\text { formação necessária aos agentes. }\end{array}$ & $\bullet$ & $\bullet$ & \\
\hline 32 & Investigação de acidentes & $\begin{array}{l}\text { Prover diretrizes e critérios para pesquisas sobre a natureza do } \\
\text { incidente, causas básicas e interpretação de cenários de riscos. }\end{array}$ & $\bullet$ & • & \\
\hline 33 & Plano de ação de emergência & $\begin{array}{l}\text { Guia de procedimentos simples para aplicação rápida de ações } \\
\text { em emergência de perigos. }\end{array}$ & & ? & \\
\hline 34 & Plano para mitigações a situações de risco & $\begin{array}{l}\text { Guia completo para reparações e mitigações em situações de } \\
\text { riscos. }\end{array}$ & $\bullet$ & $\bullet$ & \\
\hline 35 & $\begin{array}{l}\text { Revisões e reavaliação da execução de } \\
\text { planos e projetos }\end{array}$ & $\begin{array}{l}\text { Possibilidade de replanejamento das ações executadas caso } \\
\text { estejam fora dos objetivos iniciais. }\end{array}$ & & & \\
\hline 36 & Auditorias de processos & $\begin{array}{l}\text { Verificação da conformidade e efetividade dos procedimentos } \\
\text { previstos e adotados. }\end{array}$ & & $\bullet$ & \\
\hline
\end{tabular}

Analisando-se as ocorrências mais significativas no dendrograma $A$, observa-se maior similaridade de aplicação dos procedimentos nas metodologias USEPA, OMS e ATSDR nos procedimentos $31,32,1,28,25,26,15,20,9,13,3,8$ e 2, respeitando a ordem deste gráfico. Desses casos de procedimentos, sete correspondem à etapa de modelos de avaliação da exposição e toxicidade, indicando determinada uniformização nessa etapa entre as metodologias.

Os procedimentos $23,34,11,18,21$, e 12 são similares nas aplicações das metodologias USEPA e ATSDR e também correspondem à etapa $B$ de modelagem da avaliação da exposição e toxicidade, com exceção do caso 34 que corresponde ao plano para mitigações e situações de risco.

Os procedimentos $22,24,5,16,17,10,14$ e 7 são únicos de aplicação da metodologia da USEPA que se referem à etapa da exposição da avaliação e avaliação da toxicidade. Ou seja, esses procedimentos não foram observados ou foram observados parcialmente nas aplicações das outras metodologias. Sete deles referem-se à etapa B - modelos de avaliação, avaliação da exposição e toxicidade, o que significa maior aprofundamento nas investigações científicas pela metodologia da USEPA quanto à exposição de contaminantes e avaliação da toxicidade nos organismos envolvidos. Observa-se que esses pontos são únicos de aplicação da USEPA e mantêm similaridade entre si e com a etapa $A$, de identificação e especificação de riscos, pois se referem ao conhecimento do sítio contaminado, produtos nocivos e população envolvida, os quais são usados como parâmetros para a avaliação da exposição, integrando essas etapas e procedimentos. A falta de generalização desses procedimentos induz à precariedade quanto à caracterização do risco, que muitas vezes é mais quantitativa do que qualitativa, e por isso pode não despertar a atenção da população envolvida.

Os procedimentos $29,35,6$ e 27 , das etapas A e C, mostraram similaridade na falta de aplicação destes em todas as metodologias estudadas. O que caracteriza uma possível precariedade na revisão e manutenção dos procedimentos adotados na etapa de planejamento e gestão de riscos em geral. Essa situação implica em problemas de continuidade na mesma avaliação, a médio e longo prazo, principalmente nos casos de sítios contaminados, a exemplo da drenagem ácida de minas, disposição de derivados de petróleo em estuários, decomissionamento em metalúrgicas e outras. Assim, neste ponto, nos deparamos com questões relevantes em aberto, que merecem uma atenção especial nas pesquisas futuras de avaliação de risco.

Os procedimentos 4 e 19 foram únicos para USEPA e OMS. A falta dessas práticas próprias da metodologia ATSDR induz precariedade quanto ao inventário dos poluentes e 
emissões por sítios envolvidos. Possivelmente a razão disso seja motivada pelos seus objetivos institucionais vinculados a registros de doenças e efeitos à saúde.

No dendrograma $B$, pode-se observar a presença de dois clusters mostrando a similaridade entre as metodologias da ATSDR e OMS e o distanciamento com a metodologia da USEPA, devido à maior distância euclidiana. Portanto, a proximidade entre as metodologias da ATSDR e OMS é visível no gráfico mencionado e está demonstrada pelo coeficiente de correlação significativa de 0,484 , com $1 \%$ de probabilidade de erro, na Tabela 4 . Acredita-se que isso ocorre em razão da objetividade institucional que cada metodologia representa.

Com o objetivo de conhecer a existência de correlações significativas entre as variáveis correspondentes da aplicação das metodologias de avaliação de risco estudadas, aplicou-se o teste de correlação bivariada, baseado no coeficiente de correlação de intervalos de Spearman, descrito em Pestana e Gageiro (2000), onde foram utilizados os postos de dados amostrais combinados de dois a dois $(X, Y)$. Os níveis de significância foram estabelecidos em 0,01 e 0,05 em uma prova bilateral. Os resultados são mostrados na Tabela 4.

Em conformidade com esses resultados, não foram encontradas correlações extremas ou próximas a 1,0 entre as variáveis analisadas, mas podem-se considerar significativas mesmo com correlações moderadas.

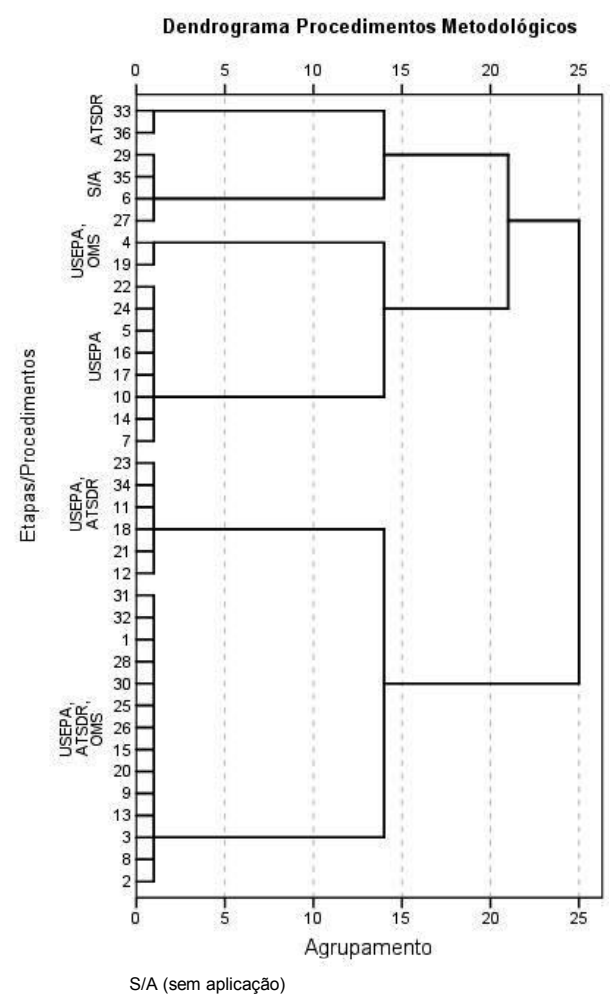

Dendrograma A

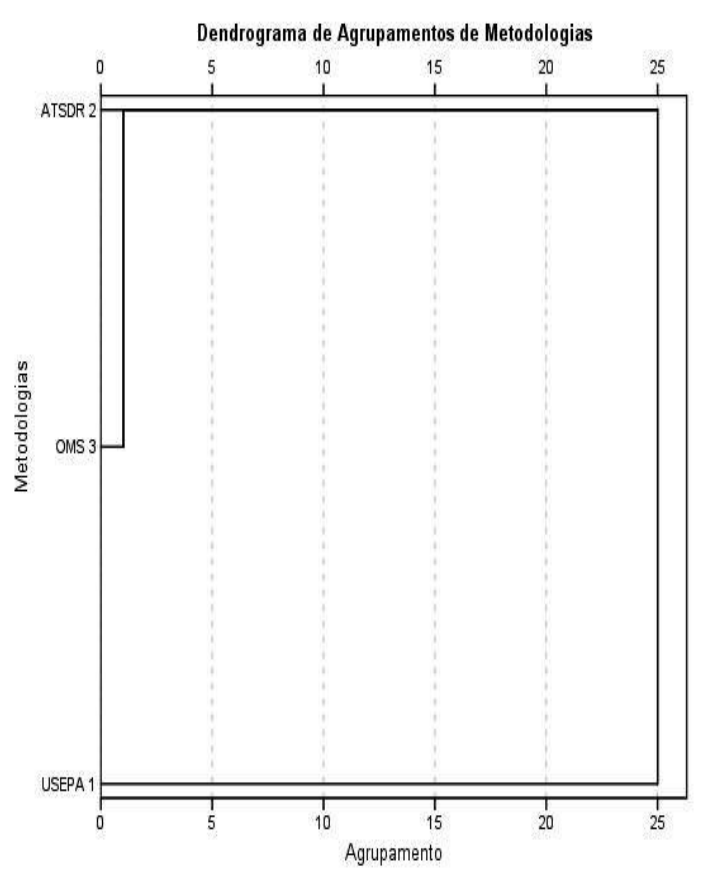

Dendrograma B

Figura 5: Dendrogramas de agrupamento hierárquico dos procedimentos aplicados por metodologia. 
Tabela 4: Correlações bivariadas através da técnica de Spearman.

\begin{tabular}{|c|c|c|c|c|c|}
\hline \multicolumn{6}{|c|}{ Correlações } \\
\hline & & & USEPA & ATSDR & OMS \\
\hline \multirow[t]{9}{*}{ Spearman's rho } & \multirow{3}{*}{ USEPA } & Coeficiente de Correlação & 1,000 & ,255 &, $400^{*}$ \\
\hline & & Sig. (p-valor) & & ,134 &, 016 \\
\hline & & $\mathrm{N}$ & 36 & 36 & 36 \\
\hline & \multirow{3}{*}{ ATSDR } & Coeficiente de Correlação & 255 & 1,000 & ,484 \\
\hline & & Sig. (p-valor) &, 134 & &, 003 \\
\hline & & $\mathrm{N}$ & 36 & 36 & 36 \\
\hline & \multirow{3}{*}{ OMS } & Coeficiente de Correlação & $400^{*}$ &, $484^{* *}$ & 1,000 \\
\hline & & Sig. (p-valor) &, 016 &, 003 & \\
\hline & & $\mathrm{N}$ & 36 & 36 & 36 \\
\hline
\end{tabular}

Este trabalho não pretende esgotar o assunto nem encerrar a questão com uma padronização metodológica. Objetiva-se indicar caminhos para estudos posteriores numa possível agregação de metodologias mais adequadas às diversas comunidades e ecossistemas. Portanto, todos os procedimentos/etapas indicados na Tabela 3 , mesmo não sendo citados em alguma metodologia, fazem parte desse contexto geral elencados na presente proposta de análise integrativa de metodologias de avaliação de risco.

\section{CONCLUSÕES}

Alguns autores têm expressado que as avaliações de riscos à saúde e ao meio ambiente tendem a ser excessivamente quantitativas e resumidas, e que elas ignoram as diferenças qualitativas. Mesmo com o conhecimento de mais de 60 métodos existentes, ainda não há uma definição padrão sobre qual metodologia é mais adequada para avaliação de riscos em determinadas situações ou de forma geral.

A análise comparativa realizada, com foco na avaliação de riscos à saúde e meio ambiente, permite encontrar as áreas melhor cobertas por cada metodologia estudada, e sugerir a metodologia complementar necessária para ser aplicada em cada caso. Observou-se que, dentre as metodologias da USEPA, ATSDR e OMS, a metodologia da USEPA é a mais citada e aplicada na prática por apresentar condições integrais ao processo de avaliação de risco, apresentando procedimentos próprios para o juízo científico tanto na avaliação da exposição quanto na avaliação dose-resposta. As metodologias da ATSDR e OMS estão mais próximas quanto à aplicação dos procedimentos, possivelmente em razão dos objetivos institucionais relacionados ao bem-estar coletivo e à saúde humana.

Nas metodologias mais utilizadas (USEPA, ATSDR e OMS), foi detectada a ausência de aspectos relevantes, relacionados com a revisão e manutenção dos procedimentos adotados, na etapa de planejamento e gestão de riscos. Esta situação compromete a continuidade na avaliação e a gestão dos riscos a médio e longo prazo. Por fim, observou-se em geral uma insuficiente caracterização qualitativa de risco e falta de mecanismos efetivos de comunicação. Estes aspectos levantados poderão incentivar e nortear a realização de trabalhos futuros que 
contemplem uma metodologia mais completa, e moldada às condições socioeconômicas e ambientais do Brasil.

\section{REFERÊNCIAS}

ATSDR. Public Health Assessment Guidance Manual, Atlanta, Georgia, 2005.

BRILHANTE, O. M.; CALDAS, L. Q. A.. Gestão de risco em saúde ambiental. FIOCRUZ, Rio de Janeiro, 2002.

CAPES. Coordenação de Aperfeiçoamento de Pessoal de Nível Superior, Out 2012.

CCPS. Guidelines for Chemical Process Quantitative risk Analysis, AIChE, New York, 1989.

CETESB. Norma Técnica P4.261. São Paulo, 2003.

CHUNG, P. W. H.; BROOMFIELD, E.; YANG, S. H.. Safety related questions for computer-controlled plants: Derivation, organization and application. Journal of Loss Prevention in the Process Industries, v.11, p.397-406, 1998.

DEMIDOVA, O.; CHERP, A.. Risk assessment for improved treatment of health considerations in EIA. Ecoline Environmental Assessment Center, Russia, p.411-429, 2005.

DOLLADILLE, O.. Proposition d'une me'thode d'analyse d'effet domino. Pre'ventique-se'curite', v.44, p.62-70, 1999.

ETA. Event tree analysis: method, graphical symbols and evaluation, Nov 1985.

IEC. 61015. Fault Tree Analysis (FTA, Out 1990.

UE - IHCP-TGD. European Commission-Joint Research Center. Techinical guidance document, 2003.

IPCS. International Programme on Chemical Safety. World Health Organization, 2013.

KENNEDY, R.; KIRWAN, B.. Development of a hazard and operability-based method for identifying safety management vulnerabilities in high risk systems. Safety Sciences, v.30, p.249-274, 1998.

KHAN, F. I.; ABBASI, S. A.. Multivariate hazard identification and ranking system. Process Safety Progress, v.17, n.3, p.157-170, 1998.

KHAN, F. I.; ABBASI, S. A.. Techniques and methodologies for risk analysis in chemical process industries. Journal of Loss Prevention in the Process Industries, v.11, p.261-277, 1998.

LIMA, C. A.. Avaliação de Risco Ambiental como Ferramenta para o Descomissionamento de uma Indústria de Metalurgia de Zinco. Tese (Doutorado em Ciências) - Universidade Federal do Rio de Janeiro, 2009.

MINISTÉRIO DA SAÚDE. Secretaria de Vigilância em Saúde, Coordenação Geral de Vigilância Ambiental em Saúde. Diretrizes Para Elaboração De Estudo De Avaliação De Risco À Saúde Humana Por Exposição A Contaminantes Químicos, Brasília, 2006.

MOITA, N. M. J.; MOITA, C. G.. Uma introdução à análise exploratória de dados multivariados. Revista Química Nova, v.21, n.4, p.467-469, 1998.

NICOLET-MONNIER, M.. Integrated regional risk assessment: The situation in Switzerland. International B Journal of Environment and Pollution, v.6, n.4-6, p.441-461, 1996.

NRC. Risk assessment in the Federal Government: managing the process. Washington, DC: National Academy Press, 1983. 
OIEN, K.; SKLET, S.; NIELSEN, L.. Development of risk level indicators for petroleum production platform. In: Proceeding from ninth international symposium loss prevention and safety promotion in the process industries, p.382-393, 1998.

OMS. Organização Mundial da Saúde, Europa: EFSA, 2006.

OMS/IPCS. Risk assessment toolkit: chemical hazards, USA, 2010.

OTA. EPA Superfund Actions and ATSDR Public, Health Data, 1995.

PESTANA, M. H.; GAGEIRO, J. N.. Análise de dados para ciências sociais: a complementaridade do SPSS. 2 ed. Lisboa: Sílabo, 2000.

PITBLADO, R. M.; WILLIAMS, J. C.; SLATER, D. H.. Quantitative assessment of process safety programs. Plant/Operations Progress, v.9, n.3, p.169-175, 1990.

ROGERS, R. L.. Methodology for the Risk Assessment of Unit Operations and Equipment for Use in Potentially Explosive Atmospheres. Library Area SAFETYNET, Inburex GmbH Hamm, 2000.

SOUZA, F. M. N.; SILVA, C. E.; AGUIAR, L. A.; ALMEIDA, J. R.. Análise de riscos como instrumento para sistemas de gestão ambiental. Revista Ibero-Americana de Ciências Ambientais, Aquidabã, v.3, n.1, p.17- 41, 2012.

TAMBELLINI A. T.; CÂMARA, V. M.. A temática saúde e ambiente no processo de desenvolvimento da Saúde Coletiva: aspectos históricos, conceituais e metodológicos. Ciência e Saúde Coletiva, v.3, n.2, p.4759, 1998.

TIXER, J.; DUSSERE, G.; SALVI, O.; GASTON, D.. Review of 62 risk analysis methodologies of industrial plants. Jornal of Loss Prevention in the Process Industries, p. 291-303, 2002.

TOOLA, A.. Plant level safety analysis. Journal of Loss Prevention in the Process Industries, v.5, n.2, p.119-124, 1992.

TROUTT, M. D.; ELSAID, H. H.. The potential value of SAATY's eigenvector scaling method for short-term forecasting of currency exchanges rates. Siam Review, v.38, p.650-654, 1996.

UE-IHCP. European Chemicals Bureau, Joint Research Centre, European Commission, Ispra, 2003.

USEPA. Risk assessment guidance for superfund. Washington, 1989.

USEPA. Ambient water quality criteria document for mercury. Prepared by the Office of Health and Environmental Assessment, Washington, 1998.

USEPA. Benchmark dose technical guidance document (BDM). Washington, 2000.

USEPA. Comparative risk framework methodology and case study. Washington, 2003.

USEPA. CatReg: categorical regression, software, user manual. Washington, 2006.

VESELY, W.E.; GOLDBERG, F. F.; ROBERTS, N. H.; HAASL, D. F.. Fault Tree Handbook. NUREG-0492 Nuclear Regulatory Commission, Washington, 1981.

VANDENBRANDE, W. W.. How to use FMEA to reduce the size of your quality toolbox. Quality Progress, v.31, n.11, p. 97-100, 1998. 